\title{
Pre Filter Hemodiafiltration with Heparin-Dose Minimization: A Safe and Effective Technique
}

\section{Sergio VO ${ }^{1 *}$, Mónica SC ${ }^{1}$, Evelyn CDR ${ }^{2}$, Raúl I' , Enzo VJ' and Héctor PGG ${ }^{1}$}

${ }^{1}$ Department of Nephrology, National Institute of Cardiology, National Autonomous University of Mexico, Mexico City, Mexico ${ }^{2}$ Department of Hematology, National Institute of Cardiology, National Autonomous University of Mexico, Mexico City, Mexico

\section{Abstract}

Background: Unfractionated heparin (UFH) administered during hemodialysis (HD) has cumulative adverse effects; hence the importance of using the lowest possible dose and avoid overdosing.

Methods: We analyzed 30 patients with chronic hemodiafiltración (HDF) treatment divided in 3 groups: pos filter HDF with heparin standard impregnation dose $(27 \pm 6.5 \mathrm{IU} / \mathrm{kg})$, HDF pos filter with impregnation low dose $(15 \pm 4.2 \mathrm{lU} /$ $\mathrm{kg}$ ); in both groups, heparin impregnation and maintenance were given, and a third group HDF pre-dilution only with heparin impregnation dose $(27 \pm 12.8 \mathrm{lU} / \mathrm{kg})$. We measured partial thromboplastin time (aPTT) and serum heparin levels at 30 and $190 \mathrm{~min}$, in the three groups.

Results: HDF pre-dilution group received a significantly lower total dose than the other 2 groups; 1750 vs. $2650 / 3444 \mathrm{UI} / \mathrm{Kg}(p<0.001)$, maintaining $30 \mathrm{~min}$ aPTT target $(55.6 \pm 43.4)$ as aPTT $190 \mathrm{~min}(44.4 \pm 30.3)$, as well as effectiveness of the treatment (substitution volume of $51.4 \pm 11.8 \mathrm{~L}$, Ktv $1.38 \pm 0.3$ ). The other two groups reached aPTT 30 min target, but decreased towards the end of HDF sessions (aPTT $190 \mathrm{~min}$ ). HDF pre-dilution did not increase clot formation $(p<0.678)$. There were no major coagulation or bleeding events in any of the HDF sessions.

Conclusion: Pre dilution HDF with only impregnation heparin is a safe and effective method; after the amplification and reproduction of our results, it could represent an alternative treatment that minimizes UFH doses in order to reduce the associated adverse events.

Keywords: Heparin; Hemodialysis; Blood; Dialysis

\section{Introduction}

Clinical nephrology practice guidelines (European, Australian, British) about required dose of UFH during hemodialysis sessions, indicate an initial loading dose, followed by a continuous infusion or maintenance boluses; these guidelines differ in doses and time of administration; in addition, for patients with increased bleeding risk, recommendations are even more varied [1-4].

Anticoagulation efficacy with UFH can be evaluated wiht aPTT or total coagulation time. It's recommended achieving an aPTT increase up to $150 \%$ than pre-dialysis values [2]. In a study published by Kessler and colleagues, they suggest measurements aPTT at the end of HD session to determine which patients benefit from reduced anticoagulation levels [5-7].

Recent evidence trends on reducing or suppressing anticoagulation dose, since patients receiving chronically UFH doses during HDF sessions, have potential cumulative adverse effects such as, endothelial dysfunction, bleeding, osteoporosis, hyperkalemia, aldosterone suppression [2].

Some groups used 50-200 ml/hr saline infusion during HD [1,6], in order to reduce or suppress anticoagulation, similar to HDF pre dilution method technique but with higher infusion volume [3].

For this reason, we analyzed patients under chronic HDF treatment, minimizing UFH dose with pre filter HDF method by suspending maintenance dose and comparing with HDF pos filter patients divided into two groups: those who received standard impregnation dose, and other whom received a low impregnation dose; both with their usual maintenance doses. We measured the levels of anticoagulation, with follow-up by levels of APTT and heparin.

\section{Materials and Methods}

Thirty prevalent ( 3 sessions per week) patients on HDF and use of UFH in each session, with no history of thrombotic events or episodes of major bleeding were included in this study. Baseline demographic and clinical characteristics are shown in Table 1. Dialysis treatment parameters are displayed in Table 2 .

\begin{tabular}{|l|l|l|l|l|}
\hline & $\begin{array}{l}\text { Pos Filter } \\
\text { Standard } \\
\text { Dose } \\
\text { N=10 }\end{array}$ & $\begin{array}{l}\text { Pos Filter } \\
\text { Low dose } \\
\mathbf{N = 1 0}\end{array}$ & $\begin{array}{l}\text { Pre Filter } \\
\text { Low Dose } \\
\text { N=10 }\end{array}$ & p value \\
\hline Age (Median \pm SD) & $44.8 \pm 7.6$ & $49.7 \pm 9.2$ & $47.7 \pm 7.8$ & ${ }^{\circ} 0.22$ \\
\hline Sex (Women/Men) & $8 / 2$ & $5 / 5$ & $5 / 5$ & ${ }^{*} 0.13$ \\
\hline Bolus UI/Kg (Median \pm SD) & $27 \pm 6.5$ & $15 \pm 4.2$ & $27 \pm 12.8$ & ${ }^{\circ} 0.02$ \\
\hline $\begin{array}{l}\text { Maintenance dose UI/Kg } \\
\text { (Median } \pm \text { SD) }\end{array}$ & $10 \pm 4.7$ & $10 \pm 8.4$ & $0 \pm 0.5$ & ${ }^{\circ} 0.024$ \\
\hline Vascular Access & & & \multirow{2}{*}{0.27} \\
\hline Catheter(N, \%) & $7(70)$ & $6(60)$ & $6(60)$ & \multirow{2}{*}{0.36} \\
\hline Fistula (N, \%) & $3(30)$ & $4(40)$ & $4(40)$ & \\
\hline Diabetes (N) & 0 & 1 & 2 & \\
\hline${ }^{*}$ Chi cuadrada; ${ }^{\circ}$ Anova & & & & \\
\hline
\end{tabular}

Table 1: Demographic and clinical characteristics of the patient population.

*Corresponding author: Sergio VO, Department of Nephrology, National Institute of Cardiology, National Autonomous University of Mexico, Mexico City, C.P. 14080, Mexico, Tel: +52 (55) 5573 2911; E-mail: sergiovao@ulm.edu.mx

Received: September 16, 2019; Accepted: October 10, 2019; Published: October 15, 2019

Citation: Sergio VO, Mónica SC, Evelyn CDR, Raúl I, Enzo VJ, et al. (2019) Pre Filter Hemodiafiltration with Heparin-Dose Minimization: A Safe and Effective Technique. J Nephrol Ther 9: 336.

Copyright: () 2019 Sergio VO, et al. This is an open-access article distributed under the terms of the Creative Commons Attribution License, which permits unrestricted use, distribution, and reproduction in any medium, provided the original author and source are credited. 


\begin{tabular}{|c|c|c|c|c|}
\hline & $\begin{array}{c}\text { Pos Filter } \\
\text { Standard Dose } \\
N=10\end{array}$ & $\begin{array}{c}\text { Pos Filter } \\
\text { Low dose } \\
\mathrm{N}=10\end{array}$ & $\begin{array}{l}\text { Pre Filter } \\
\text { Low Dose } \\
\mathrm{N}=10\end{array}$ & ${ }^{*} p$ value \\
\hline Total Dose Administered (IU) & $3444 \pm 836$ & $2650 \pm 502$ & $1750 \pm 790$ & $<0.001$ \\
\hline aTTP $30 \min (\mathrm{s})$ & $50 \pm 19.7$ & $38 \pm 5.8$ & $55.6 \pm 43.4$ & 0.372 \\
\hline aTTP $190 \mathrm{~min}(\mathrm{~s})$ & $37.2 \pm 7.1$ & $34.6 \pm 6.4$ & $44.4 \pm 30.3$ & 0.465 \\
\hline Heparin levels $30 \mathrm{~min}(\mathrm{IU} / \mathrm{mL})$ & $0.27 \pm 0.23$ & $0.11 \pm 0.12$ & $0.26 \pm 0.31$ & 0.226 \\
\hline Heparin levels $190 \mathrm{~min}(\mathrm{IU} / \mathrm{mL})$ & $0.17 \pm 0.1$ & $0.06 \pm 0$ & $0.2 \pm 0.4$ & $<0.241$ \\
\hline Basal Hemoglobin $(\mathrm{Hbi})$ & $7.6 \pm 0.9$ & $7.6 \pm 1.2$ & $9.1 \pm 1.0$ & 0.007 \\
\hline Final Hemoglobin $(\mathrm{Hbf})$ & $9.64 \pm 2.1$ & $9.4 \pm 2.2$ & $11.5 \pm 1.5$ & 0.044 \\
\hline$\Delta \mathrm{Hb}(H b f-H b i)$ & $2 \pm 1,48$ & $1.7 \pm 1.5$ & $2.4 \pm 1.18$ & 0.55 \\
\hline Coagulation score & $1.86 \pm 0.6$ & $1.88 \pm 0.3$ & $1.73 \pm 0.2$ & 0.678 \\
\hline HDF Total time (min) & $188 \pm 8.5$ & $187 \pm 4.5$ & $185 \pm 11.3$ & 0.625 \\
\hline
\end{tabular}

Table 2: Paraclinical values according to the study group.

We formed three groups of patients according to UFH doses which they had been received usualy. Pos-filter hemodiafiltration in low and high dose; likewise, patients with higher hemoconcentration were included into the HDF pre-dilution group, administering a similar dose to patients with post-high-dose HDF, but without maintenance dose.

\section{Dialytic treatment}

All HDF sessions were granted uniformly, with 5008 hemodiafiltration system of Fresenius ${ }^{\star}$ using the FxCordiax 120 filter in all cases. Blood flow higher than $300 \mathrm{ml} / \mathrm{min}$ and dialysate flow of $500 \mathrm{ml} / \mathrm{min}$ was reached in all HDF sessions. Average time was 180 minutes. Ultrafiltering was granted in adjustment according to the known dry weight.

\section{Measurements}

Values of aPTT and serum heparin (anti-Xa) levels were determined at 30 and $190 \mathrm{~min}$ ( $10 \mathrm{~min}$ after the end of the session prior to the heparinization of the lumens in the case of catheter patients), in the 3 study groups. The aPTT was considered in treatment goals when it was increased $150 \%$ (between $45-60$ seconds) according to the reference range value.

Regarding heparin levels, the treatment goal was 0.2-0.4 U/ $\mathrm{ml}$. For its measurement, we turned to the hematology department. Additionally, we record the minor and major bleeding events. Greater bleeding was defined as those events with hematocrit falling $>3 \mathrm{~g}$, hemodynamic compromise and/or transfusion requirement, and minor bleeding that auto limited or yielding with local measures.

We recorded thrombosis events by means of a visual scale, after manual irrigation with $400 \mathrm{ml}$ of saline at the end of the HD session, by two observers independently: Blood lines ( $1=$ no clot, $2=$ fibrin formation, $3=$ partial clot formation, $4=$ definitive clot formation) Dialyzer ( $1=$ clean filter, $2=$ blood streaks $<5 \%, 3=$ blood streaks $>5 \%$, $4=$ coagulated filter). Venous air trap ( $1=$ no clot, $2=$ fibrinous ring, $3=$ clot formation, $4=$ coagulation system) and we averaged the three measurements in the three groups.

\section{Results}

Within the study population, $10 \%$ of patients were carriers of diabetes mellitus. Median age was 44.3 years, $57 \%$ were female and $43 \%$ male. Within the chronic medication used, none of the patients were users of anticoagulants or anti platelets (Table 1).

Unlike patients in post-filter groups who did not maintain adequate aPTT levels at minute 190, the patients in the pre-filter group maintained aPTT in goals at both $30(55.6 \pm 43.4)$ and 190 minutes $(44.4 \pm 30.3)$ (Table 2).

To confirm this effect was due to UFH dose, we measured heparin levels at the same time (30 and 190 minutes).

The pre-HDF group maintained aPTT and heparin levels in desired ranges, while the other two groups decreased at the end of the session even at infra therapeutic ranges. The total dose received was lower with a statistically significant difference. Regarding dialytic efficacy HDF pre dilution maintained effectiveness in the treatment (substitution of 51.4 $\pm 11.8 \mathrm{~L}$, Ktv $1.38 \pm 0.3$ ) (Figure 1).

HDF pre-filter did not increase clot formation $(\mathrm{p}<0.678)$ in the dialyzer system. No thrombosis or coagulation events were recorded in any of the study groups, even when the pre-HDF group had patients with higher hemoglobin levels and the hemoconcentration that occurred after the ultrafiltrate. Additionally, there were no bleeding events, neither minor nor major in any of the study groups (Figure 2).

\section{Discussion}

Dialysis for bleeding patients or with high bleeding risk, requires special awareness during HD treatment due to potential side effects that turns it into a challenging technique for physician in every session [1].

The background of sessions with pre-dilution HDF only with UFH priming dose versus conventional hemodialysis with filter embedded in UFH, report treatment effectiveness (Ktv 1,23 \pm 0.32 ), with the need to terminate treatment in $19 \%$ of cases [7].

Bearing in mind that insufficient anticoagulation results in blood iron losses, and promotes a decrease in the purifying performance of dialysis. Anticoagulation in dialysis must be aimed at increasing efficiency during sessions [5] as recent studies showed the efficacy of heparin reduction, with a small increase in urea reduction compared to the conventional dose but taking care of preventing clotting forming $[5,6]$.

In the present study we base the efficiency of treatment on substitution objectives according to recent evidence, for convective therapies.

Results accord with saline infusion strategies, however, it had not been used with low doses of heparin in convective techniques, demonstrating dynamically that this maintains better levels of anticoagulation and efficiency of the treatment. 


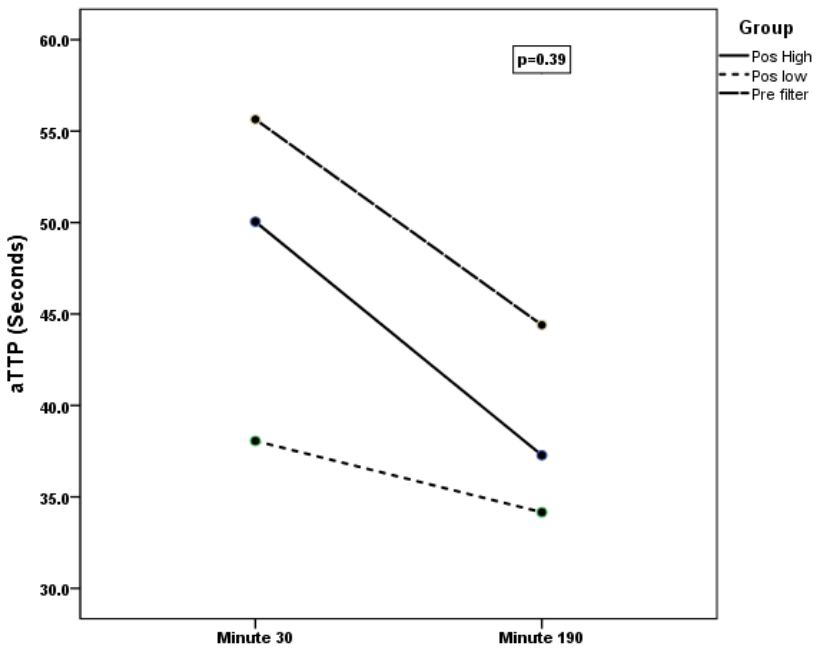

Figure 1: Comparison of aTTP values according to the study group.

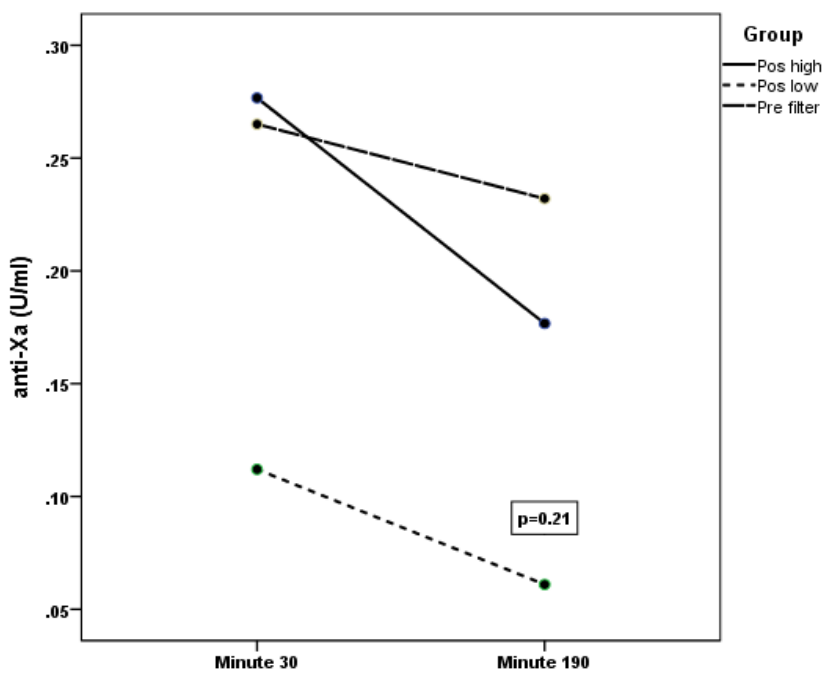

Figure 2: Comparison of anti-Xa values according to the study group.

Thisstudyhasweaknessessinceit was a single-center nonrandomized study and sample size. However, due to the physiological support and the results concordant with those of previous studies, we consider that they can be reproduced by larger studies.

We did not experience an over-clotting risk with predilutional HDF as compared to post-dilution HDF standard and low dose. Only one patient in HDF pre-dilution group, was necessary to administer 1 additional bolus of heparin, according to the doctor's evaluation during the session to avoid a major coagulation event.

\section{Conclusions}

Studies in this topic should continue, in order to have solid evidence of strategies to use fewer doses of heparin and reduce its complications, especially in patients with a high risk of bleeding in hemodialysis.

HDF pre-dilution is safe to reduce heparin dose and maintains the effectiveness of the treatment; It can even be considered to decrease the dose of UFH in subsequent studies.

\section{Statements}

\section{Statement of ethics}

Approval for the study was obtained from the Ethics Committee. Patients gave informed consent in accordance with the Declaration of Helsinki.

\section{Disclosure statement}

The authors have no conflicts of interest to declare

\section{Funding sources}

This study was financed with resources of our Institute.

\section{Author contributions}

MD Sergio Vázquez-Olvera, MD Mónica Sánchez-Cárdenas and MD Enzo Vásquez-Jiménez were responsible for the recruitment of patients and the capture of the data.

MC Evelyn Pérez Cortina, was responsible for coordinating the processing of samples and data analysis. Finally, MD Izaguirre Raúl and MD Héctor Grovas-Garza coordinated the analysis of the data as well as the writing of the manuscript.

\section{Disclosure Statement and Funding Sources}

The authors have no conflicts of interest to declare. This study was financed with resources of our Institute.

\section{References}

1. Brunot VJE, Mourad SG, Klouche K, Pernin V (2018) Heparin-free renal replacement therapy for chronic hemodialyzed patients at high risk for bleeding: a comparison of on-line predilution hemodiafiltration with conventional hemodialysis. Hemodial Int 22: 463-73.

2. European Best Practice Guidelines Expert Group on Hemodialysis, European Renal Association (2002) Section V. Chronic intermittent haemodialysis and prevention of clotting in the extracorporal system. Nephrol Dial Transplant 7: 63-71.

3. Jindal K, Chan CT, Deziel C, Hirsch D, Soroka SD, et al. (2006) Hemodialysis clinical practice guidelines for the Canadian Society of Nephrology. J Am Soc Nephrol 17: S1-27.

4. Kessler M, Moureau F, Nguyen P (2015) Anticoagulation in Chronic Hemodialysis: Progress Toward an Optimal Approach. Semin Dial 28: 474-489.

5. Mactier R, Hoenich N, Breen C (2011) Renal Association Clinical Practice Guideline on haemodialysis. Nephron Clin Pract 118: c241-286.

6. Murea M, Russell GB, Daeihagh P, Saran AM, Pandya K, et al. (2018) Efficacy and safety of low-dose heparin in hemodialysis. Hemodial Int 22: 74-81.

7. Suranyi M, Chow JS (2010) Review: anticoagulation for haemodialysis Nephrology (Carlton) 15: 386-92. 